\title{
MODELING OF CYCLIC THERMO-ELASTIC-PLASTIC BEHAVIOUR OF P91 STEEL
}

\author{
Piotr Sulich, WŁadyseaw Egner \\ Cracow University of Technology, Faculty of Mechanical Engineering, Institute of Applied Mechanics, Kraków, Poland \\ e-mail: piotrjansulich@gmail.com; wladyslaw.egner@pk.edu.pl \\ STANISEAW MrOZIŃSKI \\ UTP University of Science and Technology, Faculty of Mechanical Engineering, Bydgoszcz, Poland \\ e-mail: stanislaw.mrozinski@utp.edu.pl \\ Halina Egner \\ Cracow University of Technology, Faculty of Mechanical Engineering, Institute of Applied Mechanics, Kraków, Poland \\ e-mail: halina.egner@pk.edu.pl
}

\begin{abstract}
Thermomechanical low cycle fatigue behaviour of P91 steel used in power industry applications has been extensively investigated. The constitutive model of Armstrong-Frederick, extended with temperature rate effects, has been applied to describe the behaviour of the thermo-elastic-plastic material. The proposed model has been successfully implemented in simulation of low cycle fatigue of the examined steel in two different temperatures.
\end{abstract}

Keywords: constitutive modeling, low cycle fatigue, identification of material parameters

\section{Introduction}

Thermomechanical low cycle fatigue accompanied by elastic-plastic strains is one of the dominant failure modes in high temperature structural components such as electric power boilers, boiler pipes, engine elements, etc. Extensive research on the behaviour of various engineering materials under low cycle fatigue conditions has been carried out for the last 50 years in order to develop the adequate constitutive modeling as well as the appropriate predictions of the fatigue lifetime (Taleb et al., 2006; Zhang et al., 2008; Ganczarski and Skrzypek, 2009; Taleb and Cailletaud, 2010; Skrzypek and Ganczarski, 2015).

The aim of this paper is to work out a procedure of comprehensive analysis of thermomechanical low-cycle fatigue behaviour on the example of P91 steel, widely used in power industry applications (Duda et al., 2016). Firstly, the material is tested experimentally in two test temperatures, then the material behaviour is described by a constitutive model implemented into a numerical procedure. Next, the material characteristics are identified in different test temperatures. Finally, the numerical simulations of fatigue tests are performed and compared with the experimental results.

To reduce the number of material parameters for identification, the Armstrong and Frederick constitutive model extended with temperature rate effects is here applied.

The present work is treated as an initial step in the comprehensive analysis of P91 steel behaviour in thermomechanical fatigue conditions. For this reason, classical Armstrong and Frederick constitutive model and only two testing temperatures for parameter identification are considered. However, the procedure of analysis is general and can be applied to a more complex modeling. 


\section{Behaviour of P91 steel}

\subsection{Experimental equipment and material investigated}

Tested specimens (see Fig. 1) were cut out of a boiler pipe of diameter $d=200 \mathrm{~mm}$ and wall thickness $t=20 \mathrm{~mm}$ (see Fig. 2). The chemical composition of steel is shown in Table 1 . Low cycle fatigue tests were strain controlled with constant total strain amplitude $\left(\varepsilon_{a c}=\right.$ const , frequency of loading $0.2 \mathrm{~Hz}$ ) and constant temperature in each test.

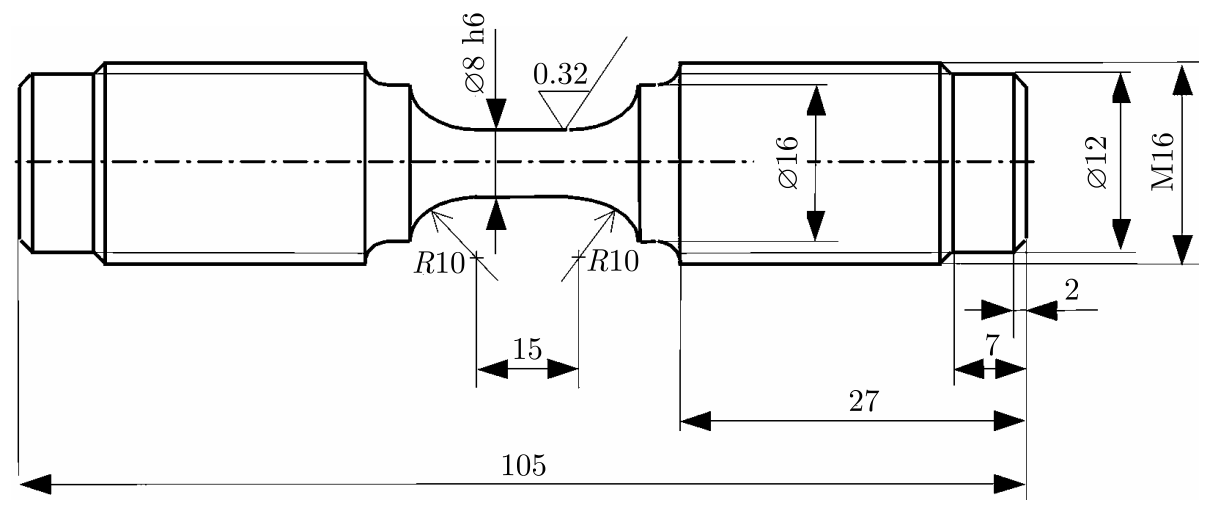

Fig. 1. Shape and dimensions of the tested specimen

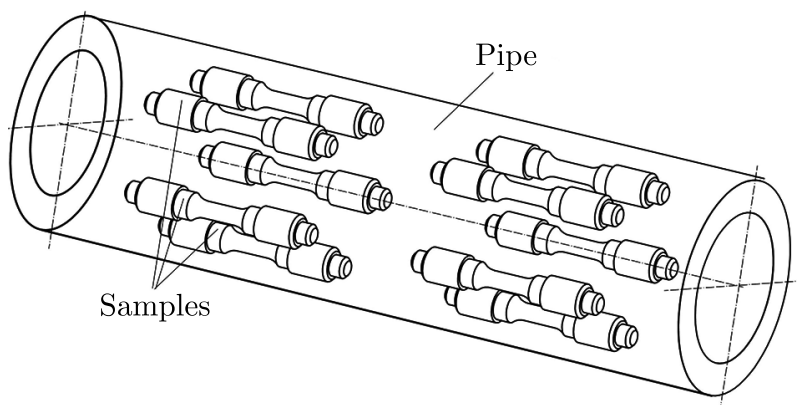

Fig. 2. Sampling procedure

Table 1. Chemical composition of the steel

\begin{tabular}{|c|c|c|c|c|c|c|c|c|c|c|c|c|c|c|}
\hline $\mathrm{C}$ & $\mathrm{Si}$ & $\mathrm{Mn}$ & $\mathrm{P}$ & $\mathrm{S}$ & $\mathrm{Cr}$ & $\mathrm{Mo}$ & $\mathrm{Ni}$ & $\mathrm{Al}$ & $\mathrm{Co}$ & $\mathrm{Cu}$ & $\mathrm{Nb}$ & $\mathrm{Ti}$ & $\mathrm{V}$ & $\mathrm{W}$ \\
\hline \hline 0.197 & 0.442 & 0.489 & 0.017 & 0.005 & 8.82 & 0.971 & 0.307 & 0.012 & 0.017 & 0.036 & 0.074 & 0.004 & 0.201 & 0.02 \\
\hline
\end{tabular}

Table 2. Loading scheme, strain amplitude and temperature

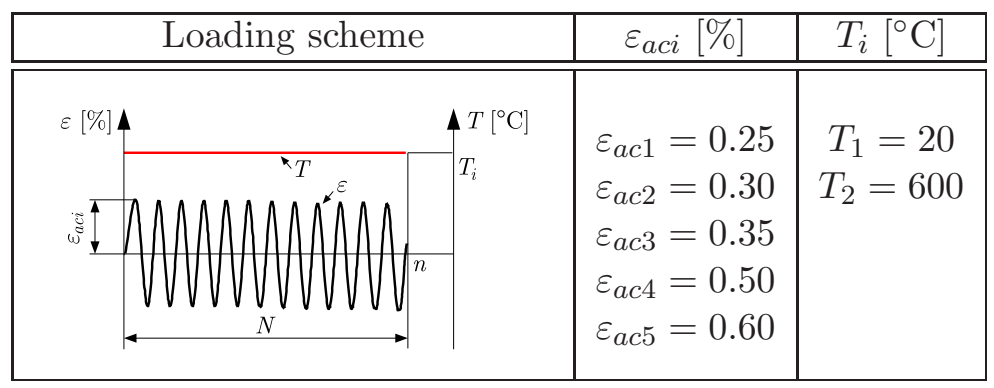

Five levels of the total strain amplitude, and two levels of temperature were applied (see Table 2). Experiments were performed on the testing machine Instron 8502 equipped with a heating chamber. 
The temperature was controlled by the use of a thermocouple attached to the sample measuring section. The material deformation was determined by means of a strain gauge extensometer (gauge length $12.5 \mathrm{~mm}$ ) (see Fig. 3).

(a)

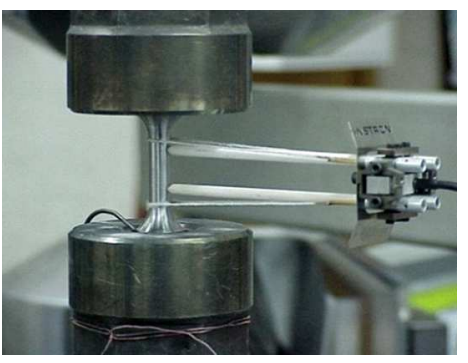

(b)

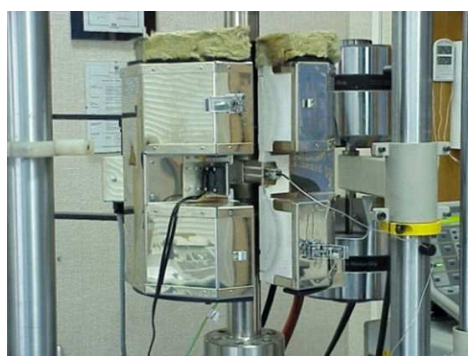

Fig. 3. The test stand: (a) ekstensometer mounting, (b) heating chamber

\subsection{Material behaviour}

The tested steel exhibits cyclic softening, regardless of the testing temperature (half-stress amplitude decreases with increasing cumulated plastic strain, cf. Golański and Mroziński (2013)). This softening could be divided into three phases, which are: the rapid softening phase during the initial few hundred cycles followed by a slow quasi-linear softening phase, and finally again fast softening till rupture (see Fig. 4) (cf. Bernhart et al., 1999; Mebarki et al., 2004). The first phase is generally explained by a rapid change in the dislocation density inherited from the quench treatment, the second is related to the formation of dislocation sub-structure and carbide coarsening under the action of time, temperature and cyclic load, while the third phase is a consequence of micro-damage development in the material that ultimately causes failure of the tested sample (cf. Seweryn et al. 2008; Szusta and Seweryn, 2010).

\section{Constitutive model of a thermo-elastic-plastic material}

\subsection{Basic assumptions}

In constitutive modeling, the well-known formalism of thermodynamics of irreversible processes with internal state variables and the local state method are often adopted (Maugin, 1999; Ottosen and Ristinmaa, 2005; Chaboche, 1997a,b, 1986; Skrzypek and Kuna-Ciskał, 2003; Egner, 2012). In this approach, we consider a material as a specific portion of the physical universe, called a system. The current state of a system is entirely determined by certain values of some independent variables, called variables of state, which can be scalars, vectors, or tensors (matrices), such as temperature (scalar) or strain (second order tensor). For a thermo-elastic-plastic material exhibiting mixed hardening the following set of state variables is defined

$$
\left\{V_{\alpha}^{s t}\right\}=\left\{\varepsilon_{i j}^{e} ; \alpha_{i j}, p ; \theta\right\}
$$

where $\varepsilon_{i j}^{e}$ are components of the reversible (elastic) strain tensor, $\alpha_{i j}$ corresponds to kinematic plastic hardening, $p$ is the accumulated plastic strain

$$
p=\int_{0}^{t} \sqrt{\frac{2}{3} \dot{\varepsilon}_{i j}^{p} \dot{\varepsilon}_{i j}^{p}} d t
$$

and $\theta$ is the absolute temperature in Kelvins. 


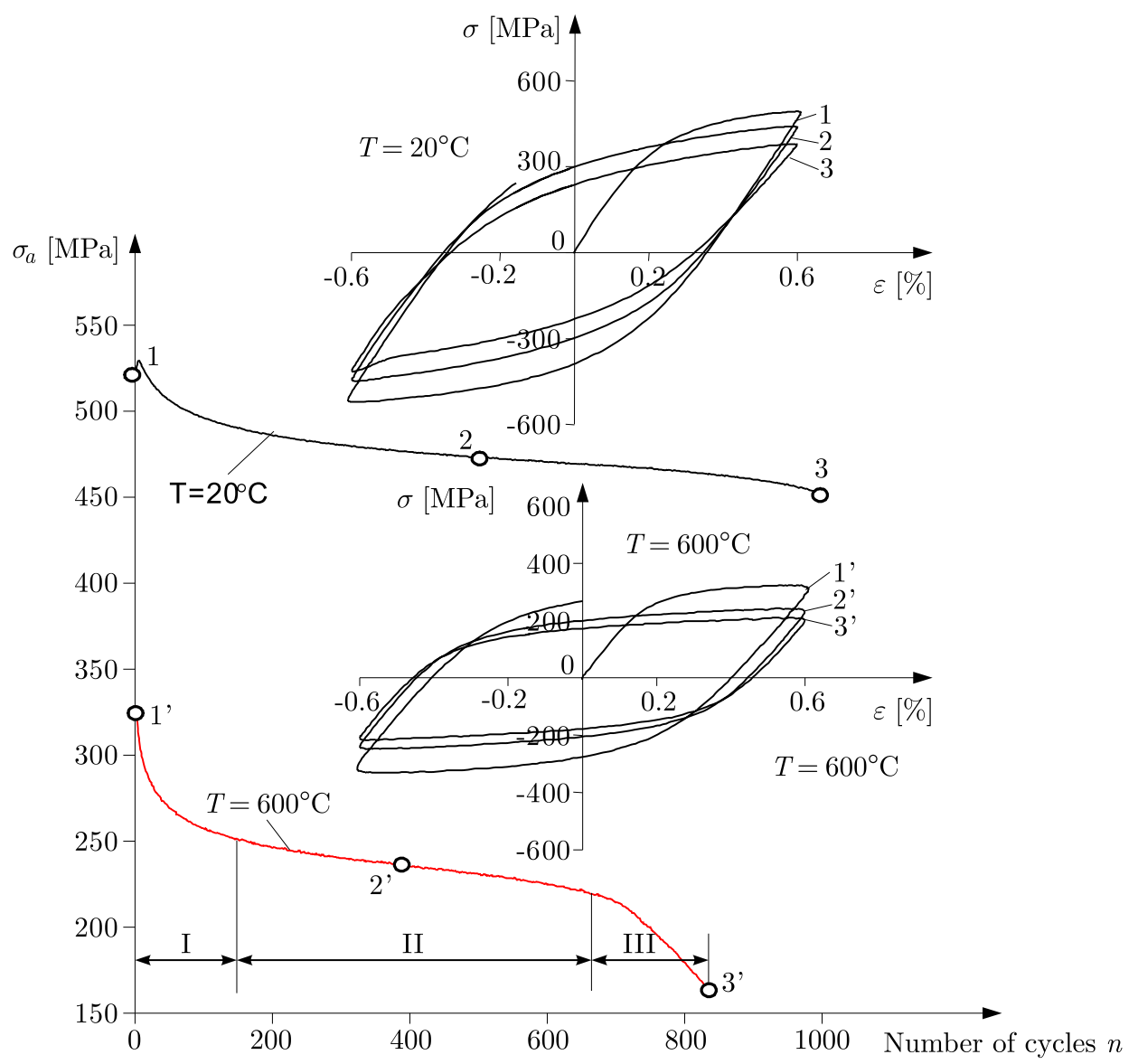

Fig. 4. Cyclic softening of the steel at a constant total strain amplitude $\varepsilon_{a c}=0.6 \%$

In the case of infinitesimal deformation, the total strain tensor components $\varepsilon_{i j}$ are expressed as the sum of the elastic (reversible) $\varepsilon_{i j}^{e}$, plastic (irreversible) $\varepsilon_{i j}^{p}$ and thermal $\varepsilon_{i j}^{\theta}$ strains

$$
\varepsilon_{i j}=\varepsilon_{i j}^{e}+\varepsilon_{i j}^{p}+\varepsilon_{i j}^{\theta}
$$

while thermal strain is expressed as

$$
\varepsilon_{i j}^{\theta}=\alpha_{i j}^{\theta}(\theta)\left(\theta-\theta_{0}\right)
$$

with $\alpha_{i j}^{\theta}(\theta)$ standing for components of the thermal expansion tensor, and $\theta_{0}$ for the reference temperature at which no thermal strains exist.

\subsection{State potential and equations of the state}

The constitutive behaviour is defined by the specification of two potentials: energy potential and dissipation potential. The state potential is a closed, convex, and scalar-valued function of the overall state variables. The Helmholtz free energy $\psi$ is here used, decomposed into thermoelastic $\rho \psi^{t e}$ and thermo-plastic $\rho \psi^{t p}$ terms ( $\rho$ is mass density)

$$
\rho \psi\left(V_{\alpha}^{s t}\right)=\rho \psi^{t e}\left(\varepsilon_{i j}^{e} ; \theta\right)+\rho \psi^{t p}\left(\alpha_{i j}, p ; \theta\right)
$$

The following classical functions are here adopted (cf. for ex. Ottosen and Ristinmaa, 2005; Egner and Egner, 2015) 


$$
\begin{aligned}
& \rho \psi^{t e}=\rho h(\theta)+\frac{1}{2}\left(\varepsilon_{i j}-\varepsilon_{i j}^{p}\right) E_{i j k l}(\theta)\left(\varepsilon_{k l}-\varepsilon_{k l}^{p}\right)-\beta_{i j}(\theta)\left(\varepsilon_{i j}-\varepsilon_{i j}^{p}\right)\left(\theta-\theta_{0}\right) \\
& \beta_{i j}=E_{i j k l}(\theta) \alpha_{k l}^{\theta}(\theta) \\
& \rho \psi^{t p}=\frac{1}{3} C(\theta) \alpha_{i j} \alpha_{i j}+Q(\theta)\left[p+\frac{1}{b(\theta)} \mathrm{e}^{-b(\theta) p}\right]
\end{aligned}
$$

where $h(\theta)$ is a function of temperature, $E_{i j k l}(\theta)$ denote components of the elastic stiffness tensor, and $C(\theta), Q(\theta), b(\theta)$ stand for temperature dependent material parameters.

The thermodynamic forces conjugated to state variables (3.1) result from the assumed form of state potential (3.5) and are defined by the following state equations

$$
\begin{aligned}
& \sigma_{i j}=E_{i j k l}(\theta)\left(\varepsilon_{k l}-\varepsilon_{k l}^{p}\right)-\beta_{i j}(\theta)\left(\theta-\theta_{0}\right) \quad X_{i j}=\frac{2}{3} C(\theta) \alpha_{i j} \\
& R=Q(\theta)\left(1-\mathrm{e}^{-b(\theta) p}\right)
\end{aligned}
$$

$\sigma_{i j}$ is here the stress tensor (thermodynamic force conjugated to elastic strain $\varepsilon_{i j}^{e}$ ), $X_{i j}$ denotes the back stress (conjugated to plastic hardening variable $\alpha_{i j}$ ), and $R$ is the drag stress (conjugated to accumulated plastic strain $p$ ).

If we now define the components of the thermodynamic conjugate force vector $\left\{J_{\alpha}\right\}$ and the flux vector components $\left\{\dot{P}_{\alpha}\right\}$ as

$$
\begin{aligned}
& \left\{J_{\alpha}\right\}=\left\{J_{\alpha}^{\text {mech }} ; J_{\alpha}^{\theta}\right\}=\left\{\sigma_{i j}, X_{i j}, R ; \frac{\theta_{, i}}{\theta}\right\} \\
& \left\{\dot{P}_{\alpha}\right\}=\left\{\dot{P}_{\alpha}^{m e c h} ; \dot{P}_{\alpha}^{\theta}\right\}=\left\{\dot{\varepsilon}_{i j}^{p},-\dot{\alpha}_{i j},-\dot{p} ;-q_{i}\right\}
\end{aligned}
$$

then the dissipation inequality can be expressed as the scalar product of $J_{\alpha}$ and $\dot{P}_{\alpha}$ as follows (Krajcinovic, 1996)

$$
\pi=J_{\alpha} \dot{P}_{\alpha} \geqslant 0
$$

where $\pi$ is the dissipation function.

\subsection{Dissipation potential and evolution equations}

A constitutive model that fulfills the Clausius-Duhem inequality fulfills all formal requirements. However, this does not guarantee that the model provides a good approximation of the real material behaviour. If the internal state variables chosen in the modeling are not identified with underlying physical mechanisms responsible for dissipation, the theory may be physically empty (Maugin, 1999). There are various approaches for the establishment of the rate laws, so that the dissipation inequality is fulfilled. The most often used is the potential approach based on the assumption of the existence of a dissipation potential $F$, being a closed, convex, and scalarvalued function of thermodynamic forces $(3.8)_{1}$, and some other possible variables. The potential of dissipation $F$ is here assumed not equal to plastic yield surface and dependent on temperature (non-associated thermo-plasticity). This allows obtaining non-linear plastic hardening rules (cf. Ganczarski et al., 2010)

$$
\begin{aligned}
& F\left(J_{\alpha}, \theta\right)=f\left(J_{\alpha}, \theta\right)+\frac{3 \gamma(\theta)}{4 C(\theta)} X_{i j}\left(\alpha_{i j}, \theta\right) X_{i j}\left(\alpha_{i j}, \theta\right) \\
& f\left(J_{\alpha}, \theta\right)=\sqrt{\frac{3}{2}\left[s_{i j}-X_{i j}\left(\alpha_{i j}, \theta\right)\right]\left[s_{i j}-X_{i j}\left(\alpha_{i j}, \theta\right)\right]}-\left[\sigma_{y}(\theta)+R(p, \theta)\right]
\end{aligned}
$$

where $f\left(J_{\alpha}, \theta\right)$ is the von Mises plastic yield surface, $s_{i j}$ is the stress deviator, $\sigma_{y}(\theta)$ denotes the yield stress, and $\gamma(\theta)$ is another temperature dependent material parameter. 
According to the generalized normality rule (cf. Chaboche, 2008), the following classical rate equations are obtained

$$
\begin{aligned}
\dot{\varepsilon}_{i j}^{p} & =\dot{\lambda}^{p} \frac{s_{i j}-X_{i j}}{\sqrt{\frac{3}{2}\left[s_{i j}-X_{i j}\left(\alpha_{i j}, \theta\right)\right]\left[s_{i j}-X_{i j}\left(\alpha_{i j}, \theta\right)\right]}} \\
\dot{\alpha}_{i j} & =\dot{\varepsilon}_{i j}^{p}-\frac{3 \gamma}{2 C} X_{i j} \dot{p} \quad \dot{p}=\dot{\lambda}^{p}
\end{aligned}
$$

The kinetic equations of force-like variables are obtained by taking time derivatives of functions $(3.7)$

$$
\dot{\sigma}_{i j}=E_{i j k l}(\theta)\left(\dot{\varepsilon}_{k l}-\dot{\varepsilon}_{k l}^{p}\right)-P_{i j} \dot{\theta}
$$

where

$$
\begin{aligned}
& P_{i j}=-\frac{\partial E_{i j k l}(\theta)}{\partial \theta}\left(\varepsilon_{k l}-\varepsilon_{k l}^{p}\right)+\frac{\partial \beta_{i j}(\theta)}{\partial \theta}\left(\theta-\theta_{0}\right)+\beta_{i j}(\theta) \\
& \dot{X}_{i j}=\frac{2}{3} C(\theta) \dot{\alpha}_{i j}+\frac{2}{3} \frac{d C(\theta)}{d \theta} \alpha_{i j} \dot{\theta} \\
& \dot{R}=Q(\theta) b(\theta) \mathrm{e}^{-b(\theta) p} \dot{p}+\left[\frac{d Q(\theta)}{d \theta}\left(1-\mathrm{e}^{-b(\theta) p}\right)+Q(\theta) \frac{d b(\theta)}{d \theta} \mathrm{e}^{-b(\theta) p} p\right] \dot{\theta}
\end{aligned}
$$

\subsection{Consistency condition}

Calculation of the Lagrange multiplier $\dot{\lambda}^{p}(\theta)$ for rate-independent material needs making use of the consistency condition

$$
\dot{f}\left(J_{\alpha}, \theta\right)=\frac{\partial f}{\partial \sigma_{i j}} \dot{\sigma}_{i j}+\frac{\partial f}{\partial X_{i j}} \dot{X}_{i j}+\frac{\partial f}{\partial R} \dot{R}+\frac{\partial f}{\partial \theta} \dot{\theta}=0
$$

The classical Kuhn-Tucker loading/unloading conditions have the form

$$
f \leqslant 0 \text { and } \dot{f}\left\{\begin{array}{llll}
<0 & \text { and } & \dot{\lambda}^{p}=0 \Rightarrow \text { passive loading } \\
=0 & \text { and } & \dot{\lambda}^{p}=0 \Rightarrow & \text { neutral loading } \\
=0 & \text { and } & \dot{\lambda}^{p}>0 & \Rightarrow \text { active loading }
\end{array}\right.
$$

Substituting equations (3.7) and (3.11) into equation (3.14) leads to the following

$$
\begin{aligned}
\dot{f}= & \frac{\partial f}{\partial \sigma_{i j}} \dot{\sigma}_{i j}-\dot{\lambda}^{p}\left[\frac{2}{3} C \frac{\partial f}{\partial \sigma_{i j}}\left(\frac{\partial f}{\partial \sigma_{i j}}-\gamma \alpha_{i j}\right)-\frac{\partial f}{\partial R} Q b \mathrm{e}^{-b p}\right]-\left\{\frac{2}{3} \frac{d C}{d \theta} \frac{\partial f}{\partial \sigma_{i j}} \alpha_{i j}\right. \\
& \left.-\frac{\partial f}{\partial R}\left[\frac{d Q}{d \theta}\left(1-\mathrm{e}^{-b p}\right)+Q \frac{d b}{d \theta} \mathrm{e}^{-b p} p\right]-\frac{\partial f}{\partial \theta}\right\} \dot{\theta}=\frac{\partial f}{\partial \sigma_{i j}} \dot{\sigma}_{i j}-\dot{\lambda}^{p} H-\dot{\theta} S=0
\end{aligned}
$$

In the above equation, $H$ is the generalized hardening modulus

$$
H=\frac{2}{3} C \frac{\partial f}{\partial \sigma_{i j}}\left(\frac{\partial f}{\partial \sigma_{i j}}-\gamma \alpha_{i j}\right)-\frac{\partial f}{\partial R} Q b \mathrm{e}^{-b p}
$$

and $S$ reflects the sensitivity of the yield surface on temperature changes (cf. Egner, 2012)

$$
S=\frac{2}{3} \frac{d C}{d \theta} \frac{\partial f}{\partial \sigma_{i j}} \alpha_{i j}-\frac{\partial f}{\partial R}\left[\frac{d Q}{d \theta}\left(1-\mathrm{e}^{-b p}\right)+Q \frac{d b}{d \theta} \mathrm{e}^{-b p} p\right]-\frac{\partial f}{\partial \theta}
$$

Expression (3.16) determines the consistency multiplier

$$
\dot{\lambda}^{p}(\theta)=\frac{1}{w}\left[\frac{\partial f}{\partial \sigma_{i j}} E_{i j k l} \dot{\varepsilon}_{k l}-\left(\frac{\partial f}{\partial \sigma_{i j}} P_{i j}+S\right) \dot{\theta}\right]
$$

where

$$
w=\frac{\partial f}{\partial \sigma_{i j}} E_{i j k l} \frac{\partial f}{\partial \sigma_{k l}}+H
$$




\subsection{Heat balance equation}

To determine the temperature distribution within the body, the heat balance equation is used, derived from the first law of thermodynamics by substituting into it the internal energy density together with Fourier's law. The heat balance equation takes the form $\left(c_{\varepsilon}^{\theta}\right.$ is the specific heat capacity at a constant strain and $r$ is the distributed heat source per unit volume)

$$
\rho c_{\varepsilon}^{\theta} \dot{\theta}=-q_{i, i}+r-\theta P_{i j}\left(\dot{\varepsilon}-\dot{\varepsilon}_{i j}^{I}\right)+\sigma_{i j} \dot{\varepsilon}_{i j}^{p}-\left(R-\theta \frac{\partial R}{\partial \theta}\right) \dot{p}-\left(X_{i j}-\theta \frac{\partial X_{i j}}{\partial \theta}\right) \dot{\alpha}_{i j}
$$

\section{Numerical implementation and results}

\subsection{Numerical algorithm}

In the case of uniaxial loading (tension/compression), the tensorial quantities: stress tensor, strain tensor and back stress tensor may be presented in the following matrix forms

$$
\begin{aligned}
& {\left[\sigma_{i j}\right]=\sigma\left[\begin{array}{lll}
1 & 0 & 0 \\
0 & 0 & 0 \\
0 & 0 & 0
\end{array}\right] \quad\left[s_{i j}\right]=\sigma\left[\begin{array}{ccc}
\frac{2}{3} & 0 & 0 \\
0 & -\frac{1}{3} & 0 \\
0 & 0 & -\frac{1}{3}
\end{array}\right]} \\
& {\left[\varepsilon_{i j}\right]=\varepsilon^{e}\left[\begin{array}{ccc}
1 & 0 & 0 \\
0 & -\nu & 0 \\
0 & 0 & -\nu
\end{array}\right]+\varepsilon^{p}\left[\begin{array}{ccc}
1 & 0 & 0 \\
0 & -\frac{1}{2} & 0 \\
0 & 0 & -\frac{1}{2}
\end{array}\right]+\alpha^{\theta}\left(\theta-\theta_{0}\right)\left[\begin{array}{ccc}
1 & 0 & 0 \\
0 & 1 & 0 \\
0 & 0 & 1
\end{array}\right]} \\
& {\left[X_{i j}\right]=X\left[\begin{array}{ccc}
\frac{2}{3} & 0 & 0 \\
0 & -\frac{1}{3} & 0 \\
0 & 0 & -\frac{1}{3}
\end{array}\right] \quad\left[\alpha_{i j}\right]=\alpha\left[\begin{array}{ccc}
1 & 0 & 0 \\
0 & -\frac{1}{2} & 0 \\
0 & 0 & -\frac{1}{2}
\end{array}\right]}
\end{aligned}
$$

The numerical procedure implementing EAF model has been built by the use of Mathematica 10 software according to the algorithms based on the classical backward Euler scheme and the Newton-Raphson method (cf. Chaboche and Cailletaud, 1996) (see Fig. 5). The vector $\Delta S=$ $\left[\Delta \lambda, \Delta \varepsilon^{e}, \Delta \theta, \Delta \alpha\right]^{\mathrm{T}}$ contains increments of the unknowns, and is iteratively calculated according to

$$
\Delta S_{(k+1)}=\Delta S_{(k)}-\left[J_{(k)}\right]^{-1} R\left(\Delta S_{(k)}\right)
$$

In the above equation $[J]=\partial R / \partial \Delta S$ is the Jacobian matrix and $R(\Delta S)$ is the residual vector containing the components $R_{i}=\Delta S_{i}-\widehat{\Delta S}_{i}$, where $\Delta S_{i}$ is a variable while $\widehat{\Delta S}_{i}$ denotes the function resulting from the evolution rule for the $i$-th variable $S_{i}$. It is evident that the condition $R(\Delta S)=0$ defines the solution. If we expand this condition into a Taylor series, we obtain (4.2). The iteration procedure is stopped when the norm of $R$ is sufficiently small.

\subsection{Identification of model parameters}

The identification of model parameters has been performed with the application of SIMULIA-Isight package (cf. SIMULIA Abaqus Extended Products, 2014), which provides a platform for automatic optimal selection of material parameters. For this purpose, two components offered by the program: "Data Matching" and "Optimization" have been used. "Data Matching" component allows one to calibrate the model by analyzing different error measures between the experimental data and numerical simulation results. The following vector of material parameters $P_{i}$ is searched

$$
\left\{P_{i}\right\}=\left\{\sigma_{y}, E, \gamma, C, b, Q\right\} \quad P_{i} \in\left\langle L_{i}, U_{i}\right\rangle
$$




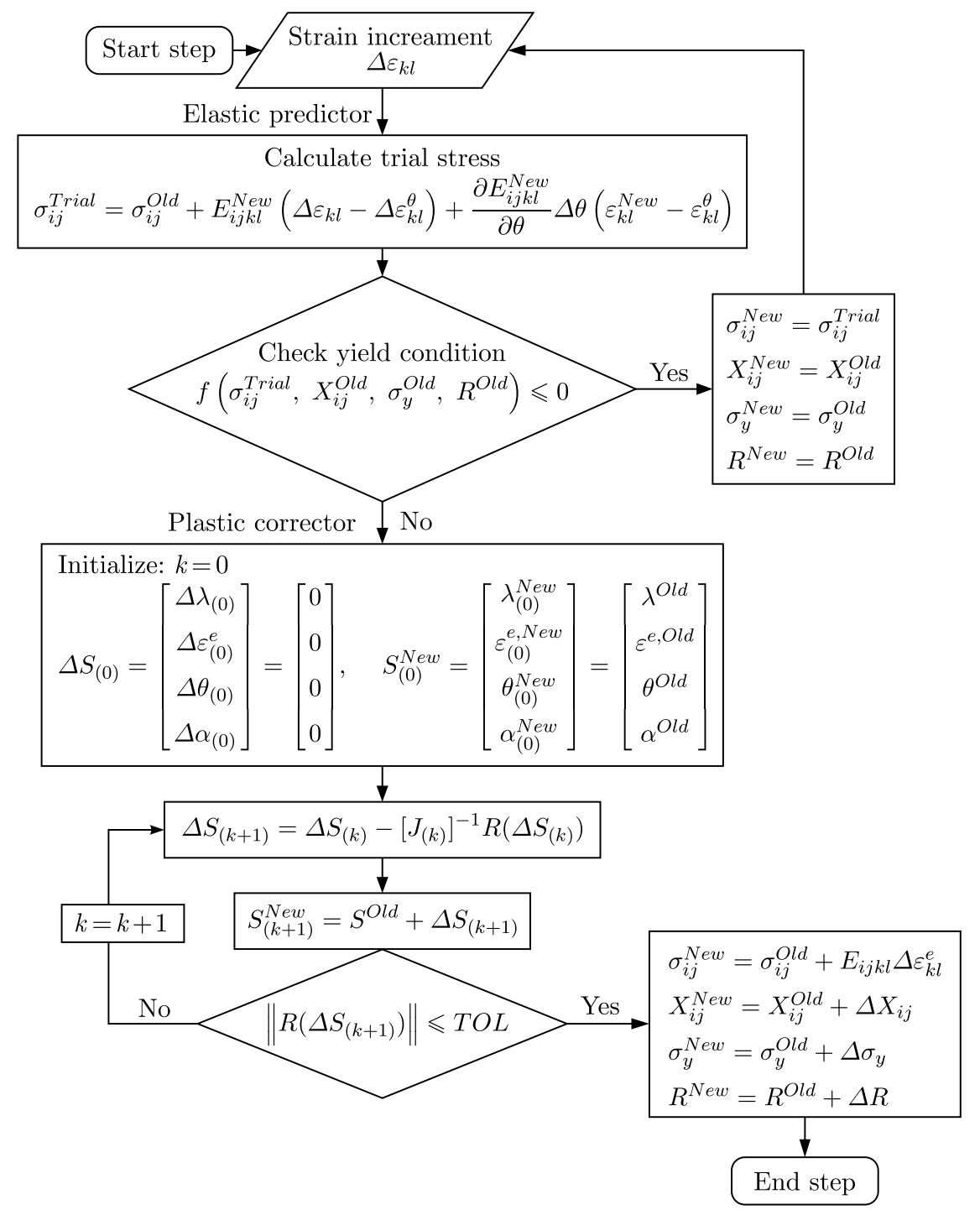

Fig. 5. Numerical algorithm

The parameters are bounded between their respective lower bounds $L_{i}$ and upper bounds $U_{i}$. Before the identification starts, the parameters are subjected to normalization, so that

$$
\bar{P}_{i} \in\langle-1,1+\rangle \quad P_{i}=\frac{1}{2}\left[\left(1-\bar{P}_{i}\right) L_{i}+\left(1+\bar{P}_{i}\right) U_{i}\right]
$$

In the present analysis, the error measure used is the square root of the sum of squared deviations

$$
F_{o b j}\left(\bar{P}_{i}\right)=\sum_{k=1}^{m}\left[\sigma_{k}\left(P_{i}\right)-\sigma_{k}^{e}\right]^{2}
$$

where $\sigma_{k}^{e}$ denote the experimental stress data and $\sigma_{k}\left(P_{i}\right)$ are the stress data calculated numerically by the use of current values of the model parameters $P_{i}$.

The "optimization" component is applied to find an optimal solution in the user-defined field, with the assumed constraints and the objective function defined by (4.5). The constraints limit the search field to the range of acceptable physical values. To reduce the dimension of the field in which the optimal solution is searched, the following procedure has been applied: the initial yield stress $\sigma_{y}$ and the elastic modulus $E$ are identified manually, considering the 
monotonic tensile part of the first hysteresis loop. Next, considering the whole first hysteresis loop, the approximate values of kinematic hardening parameters $(\gamma$ and $C$ ) are looked for on the assumption that the isotropic hardening is negligible $(b=0$ and $Q=0)$

$$
\min \left\{F_{o b j}\left(\bar{P}_{i}\right): \quad E=\text { const } \wedge \sigma_{y}=\text { const } \wedge b=0 \wedge Q=0\right\}
$$

Then the parameters related to the isotropic hardening are searched with the use of several chosen hysteresis loops (loop 2 to 20)

$$
\min \left\{F_{o b j}\left(\bar{P}_{i}\right): E=\text { const } \wedge \sigma_{y}=\text { const } \wedge \gamma=\text { const } \wedge C=\text { const }\right\}
$$

As a result, the approximate values of material parameters, constituting the starting point for optimization are set, and the identification of all the unknown material parameters $\left(E, \sigma_{y}, \gamma, C, b, Q\right)$ is performed once again, but in a substantially limited range around the initial point. The results of the applied identification procedure are presented in Table 3. Please note that these data ensure the best fit of the results simulated numerically into the experimental results for the chosen objective function $F_{o b j}\left(\bar{P}_{i}\right)$. However, the constitutive model does not account for nonlinear elastic effects, therefore the yield stress is clearly underestimated relative to the offset yield stress $R_{p 02}$ for the considered material (cf. Mroziński and Piotrowski, 2013).

Table 3. Material parameters $\left(\varepsilon_{a c}=0.60 \%\right)$

\begin{tabular}{|l|c|c|}
\cline { 2 - 3 } \multicolumn{1}{c|}{} & $20^{\circ} \mathrm{C}$ & $600^{\circ} \mathrm{C}$ \\
\hline \hline$\sigma_{y}[\mathrm{MPa}]$ & 278 & 184 \\
\hline$E[\mathrm{MPa}]$ & 198000 & 159000 \\
\hline$\gamma$ & 595 & 752 \\
\hline$C[\mathrm{MPa}]$ & 130420 & 89120 \\
\hline$b$ & 1.02 & 1.88 \\
\hline$Q[\mathrm{MPa}]$ & -39 & -69 \\
\hline
\end{tabular}

The experimental observations indicate that steel is not stable during fatigue, and that the microstructure can be modified by the thermal cycle. However, such a case takes place when temperatures reach or exceed the tempering temperatures, even for a short time (cf. Zhang et al., 2008). In other words, there are two ranges of temperature, in which the fatigue behaviour of the steel is different:

(1) Above the tempering temperature, a sharp ageing is observed while the fatigue test additionally enhances the microstructural evolutions related to ageing. In such a case, changes of mechanical properties of the steel are induced by factors related independently to the history of temperature. As a consequence, it is necessary to include additional parameters (state variables) for correct description of such an influence of temperature in a constitutive model. The identification of model parameters, being temperature history dependent, should be done for all temperatures by identifying with all tests together with the chosen material functions of temperature (cf. Cailletaud et al., 2000). For this purpose, an experimental thermomechanical fatigue tests should be performed and used for parameter identification.

(2) On the other hand, below the tempering temperature (the case considered in the present analysis) the ageing remains nearly constant, so that the mechanical properties depend on the current temperature and not on the history of temperature. In such a case, there is no need to include in the identification procedure all temperatures together, but to use several isothermal tests in different temperatures, and introduce the influence of temperature on the material parameters by interpolation techniques with polynomial or spline functions. 


\subsection{Simulation of the tests under strain control}

Kinematic hardening equations $(3.11)_{2}$ and $(3.13)_{2}$, proposed by Armstrong and Frederick (1996), introduce a recall term, which is collinear with the back stress $X_{i j}$. For a monotonic uniaxial loading, the evolution of $X_{i j}$ becomes exponential with a saturation value $C / \gamma$. Integration of $(3.13)_{2}$ with respect to $\varepsilon_{i j}^{p}$ (for the isothermal case) yields

$$
X_{i j}=\nu \frac{C}{\gamma}\left(1-\mathrm{e}^{-\nu \gamma \varepsilon_{i j}^{p}}\right)
$$

where $\nu= \pm 1$ indicates the flow direction. Function (4.8) is shown in Fig. 6a for two testing temperatures. It can be seen that the translation of the yield surface for the same strain level is more pronounced in lower temperatures.

(a)

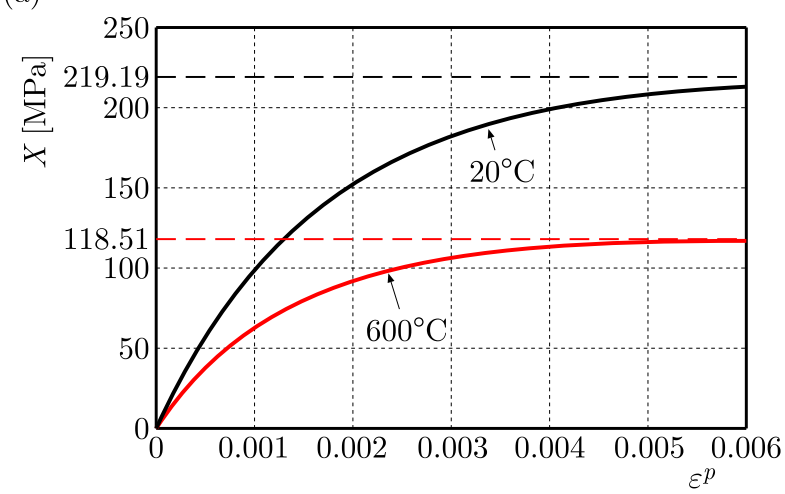

(b)

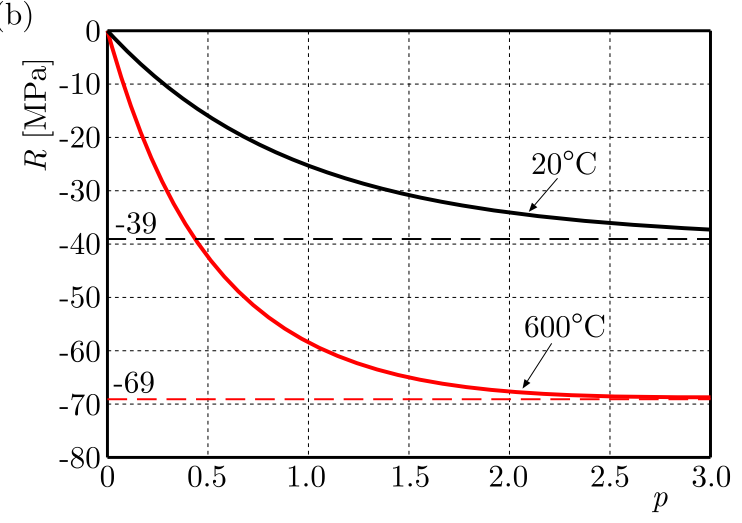

Fig. 6. (a) Evolution of back stress versus plastic strain. (b) Evolution of $R$ versus accumulated plastic strain

The $R$ - $p$ curve obtained on the basis of Eq. (3.7) 3 is shown in Fig. 6b. The parameters $Q$ and $b$ depend on the material and temperature. For cyclic loading, the value of $b$ is usually placed in the range between 0.5 and 50 . In the context of monotonic loading, the value of $b$ should be much higher (cf. Chaboche, 2008).

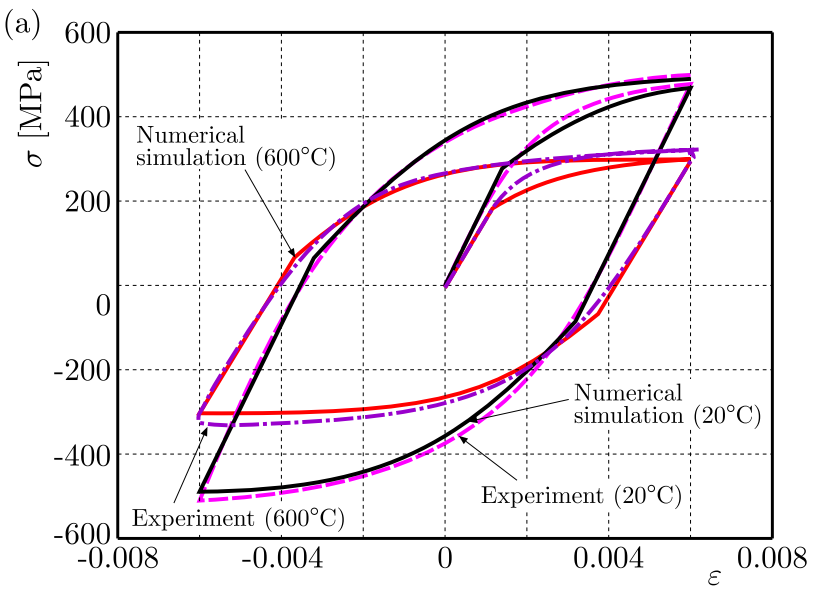

(b)

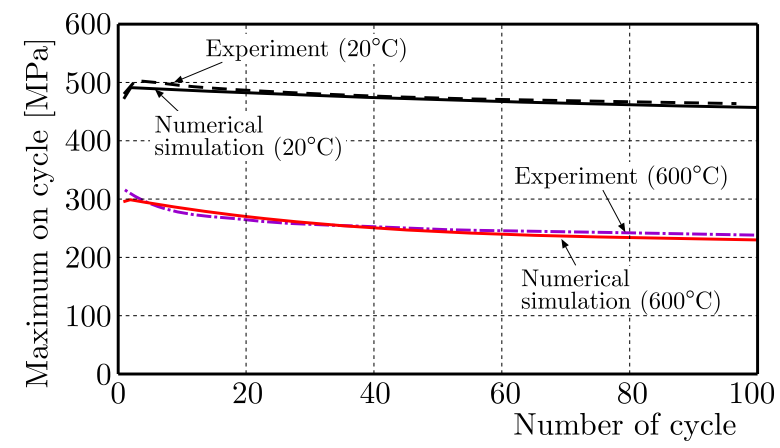

Fig. 7. Comparison between experimental and calculated (a) responses with a strain rate of $10^{-2}$ and (b) maximum stress vs. cycles at temperatures of $20^{\circ} \mathrm{C}$ and $600^{\circ} \mathrm{C}$

Figure 7a presents a comparison between the test results used in the identification process and their simulations by the described model using the material parameters for Table 3. As shown in Fig. 7a, the model simulates the first hysteresis loop with a very good accuracy. Also, stage I of the tests used in the identification process (the rapid softening phase during the initial few hundred cycles) is well reflected, see Fig. 7b. 
The identification is performed with the use of chosen hysteresis loops only, and not with the whole fatigue curve. However, when the optimal values of model parameters are obtained, the (numerically obtained) maximum stress on cycle versus cycle number exhibits a good agreement with the results of all the first 100 cycles of the experiment (see Fig. 7b).

\section{Conclusions}

In the present paper, the algorithm for a comprehensive analysis of thermomechanical fatigue behaviour is presented. Such an analysis consists of five main steps: (1) experimental testing in several test temperatures, (2) constitutive modeling of material behaviour regarding the efects of temperature change, (3) numerical implementation of the mathematical model, (4) identification of model parameters in different test temperatures to obtain temperature-dependent material characteristics, and (5) validation of the analysis by comparison between the experimental and numerical results.

The classical Armstrong and Frederick constitutive model used in the presented analysis is not capable of describing different physical mechanisms able to produce material nonlinearities on a macro-scale. For this reason, a more advanced constitutive model should be adopted to properly refelect the material behaviour under different loading paths (cf. Besson et al., 2009; Saanouni, 2012; Egner and Egner, 2014). However, such advanced models involve many material parameters that need to be identified. This is why in this research a simple model is considered, but the described procedure is general and can be applied also to a more compehensive modeling (cf. Velay et al., 2006; Saï, 2011).

\section{References}

1. Armstrong P.J., Frederick C.O., 1966, A mathematical representation of the multiaxial Bauschinger effect, Report RD/B/N731, CEGB, Central Electricity Generating Board, Berkeley, UK

2. Bernhart G., Moulinier G., Brucelle O., Delagnes D., 1999, High temperature low cycle fatigue behaviour of a martensite forging tool steel, International Journal of Fatigue, 21, 2, 179-186

3. Besson J., Cailletaud G., Chaboche J.L., Forest S., Blétry M., 2009, Non-Linear Mechanics of Materials, Springer

4. Снавосне J.L., 1997a, Thermodynamic formulation of constitutive equations and application to the viscoplasticity and viscoelasticity of metals and polymers, International Journal of Solids and Structures, 34, 18, 2239-2254

5. Chaвоche J.L., 1997b, Viscoplastic constitutive equations for the description of cyclic and anisotropic behaviour of metals, Bulletin de L'Academie Polonaise des Sciences, Série des Sciences Techniques, XXV , 1, 33-39

6. Снавосне J., 1986, Time independent constitutive theories for cyclic plasticity, International Journal of Plasticity, 2, 2, 149-188

7. Снавосне J.L., 2008, A review of some plasticity and viscoplasticity constitutive theories, International Journal of Plasticity, 24, 1642-1693

8. Chaboche J.L., Cailletaud, G., 1996, Integration methods for complex plastic constitutive equations, Computer Methods in Applied Mechanics and Engineering, 133, 125-155

9. Cailletaud G., Depoid C., Massinon D., Nicouleau-Bourles E., 2000, Elastoviscoplasticity with aging in aluminium alloys, [In:] Continuum Thermomechanics: The Art and Science of Modelling Material Behaviour (Paul Germain's Anniversary Volume), Solid Mechanics and Its Applications, Kluwer Academic Publishers, 75-86

10. Duda P., Felkowski Ł., Dobrzański J., Purzyńska H., 2016, Modelling the strain and stress state under creep conditions in P91 steel, Materials at High Temperatures, 33, 85-93 
11. EgNer H., 2012, On the full coupling between thermo-plasticity and thermo-damage in thermodynamic modeling of dissipative materials, International Journal of Solids and Structures, 49, 279-288

12. Egner H., Egner W., 2015, Classification of constitutive equations for dissipative materialsgeneral review, [In:] Mechanics of Anisotropic Materials, J.J. Skrzypek and A.W. Ganczarski (Edit.), 247-294, Springer

13. Egner H., EGNER W., 2014, Modeling of a tempered martensitic hot work tool steel behavior in the presence of thermo-viscoplastic coupling, International Journal of Plasticity, 57, 77-91

14. Ganczarski A.W., Egner H., Muc A., Skrzypek J.J., 2010, Constitutive models for analysis and design of multifunctional technological materials, [In:] Innovative Technological Materials. Structural Properties by Neutrons, Synchrotron Radiation and Modelling, F. Rustichelli and J.J. Skrzypek (Edit.), 179-220, Springer

15. Ganczarski A., Skrzypek J., 2009, A study on coupled thermo-elasto-plastic-damage dissipative phenomena: models and application to some innovative materials, Journal of Thermal Stresses, 32, 698-751

16. Golański G., Mroziński S., 2013, Low cycle fatigue and cyclic softening behavior of martensitic cast steel, Engineering Failure Analysis, 12, 35, 692-702

17. Krajcinovic D., 1996, Damage Mechanics, Elsevier

18. Maugin G. A., 1999, The Thermomechanics of Nonlinear Irreversible Behaviors. An Introduction, World Scientific Series on Nonlinear Science, Series A, Vol. 27

19. Mebarki N., Delagnes D., Lamesle P., Delmas F., Levaillant C., 2004, Relationship between microstructure and mechanical properties of a $5 \% \mathrm{Cr}$ tempered martensitic tool steel, Materials Science and Engineering A, 387-389, 1/2, 171-175

20. Mroziński S., Piotrowski M., 2013, Influence of temperature and loading program on the fatigue life of steel P91, Acta Mechanica et Automatica, 7, 2, 93-98

21. Ottosen N. S., RistinmaA M., 2005, The Mechanics of Constitutive Modeling, Elsevier

22. SaAnouni, K., 2012, Damage Mechanics in Metal Forming: Advanced Modeling and Numerical Simulation, ISTE/Wiley, London.

23. SAÏ K., 2011, Multi-mechanism models: Present state and future trends, International Journal of Plasticity, 27, 250-281

24. Seweryn A., Buczyński A., Szusta J., 2008, Damage accumulation model for low cycle fatigue, International Journal of Fatigue, 30, 756-765

25. SIMULIA Abaqus Extended Products, Abaqus 6.14-AP Isight 5.9, Dassault Systemes 1994-2014, http://www.3ds.com/products/simulia

26. Skrzypek J., Ganczarski A., 2015, Mechanics of Anisotropic Materials, Springer

27. Skrzypek J., KunA-Ciską H., 2003, Anisotropic elastic-brittle-damage and fracture models based on irreversible thermodynamics, [In:] Anisotropic Behaviour of Damaged Materials, J. Skrzypek and A. Ganczarski (Edit.), Lecture Notes in Applied and Computational Mechanics, vol. 9, 143-184, Springer

28. Szusta J., Seweryn A., 2010, Low-cycle fatigue model of damage accumulation - The strain approach, Engineering Fracture Mechanics, 77, 1604-1616

29. Taleb L., Cailletaud G., 2010, An updated version of the multimechanism model for cyclic plasticity, International Journal of Plasticity, 26, 859-874

30. Taleb L., Cailletaud G., Blaj L., 2006, Numerical simulation of complex ratcheting tests with a multi-mechanism model type, International Journal of Plasticity, 22, 724-753

31. Velay V., Bernhart G., Penazzi L., 2006, Cyclic behavior modeling of a tempered martensitic hot work tool steel, International Journal of Plasticity, 22, 459-496

32. Zhang Z., Bernhart G., Delagnes D., 2008, Cyclic behavior constitutive modeling of a tempered martensitic steel including ageing effect, International Journal of Fatigue, 30, 706-716 